\title{
A new approach to (quasi) periodic boundary conditions in micromagnetics: the macro geometry
}

\author{
Hans Fangohr, ${ }^{1}$ Giuliano Bordignon, ${ }^{1,2}$, Matteo Franchin, ${ }^{1,2}$ \\ Andreas Knittel, ${ }^{1}$ Peter A. J. de Groot, ${ }^{2}$ Thomas Fischbacher ${ }^{1}$ \\ ${ }^{1}$ School of Engineering Sciences, ${ }^{2}$ School of Physics and Astronomy, \\ University of Southampton, SO17 1BJ Southampton, United Kingdom
}

\begin{abstract}
We present a new method to simulate repetitive ferromagnetic structures. This macro geometry approach combines treatment of short-range interactions (i.e. the exchange field) as for periodic boundary conditions with a specification of the arrangement of copies of the primary simulation cell in order to correctly include effects of the demagnetizing field. This method (i) solves a consistency problem that prevents the naive application of $3 \mathrm{~d}$ periodic boundary conditions in micromagnetism and (ii) is well suited for the efficient simulation of repetitive systems of any size.
\end{abstract}

Introduction One often wants to quantitatively understand the behaviour of highly regular extended periodic magnetic structures created, for example, by selfassembly methods. As these structures often are too large for full micromagnetic simulation, some physically justifiable assumptions have to be made to reduce the computational complexity.

One attractive simplification is to define a 'primary' simulation cell $C_{0}$ in the bulk of the sample (which may contain one or multiple elementary lattice cells) and demand that the magnetization in every other 'image' cell $C_{j}$ is constrained to behaving as a translated copy of the magnetization in cell $C_{0}$. While this approximation loses long range multicell structures (such as the domain wall structure of extended materials) and field deviations near surfaces, it is useful in other situations such as magnetic films in strong external fields.

This idea is usually described by the term 'periodic boundary conditions' (PBC). We note that there is a conceptual difference between using PBC to remove surface artefacts in multi-particle statistical mechanics simulations (e.g. of a gas of hard spheres) and using PBC to describe real periodic structures with long-range interactions (such as magnetic repulsion).

While no problem arises for structures that are periodic in one or two directions only, micromagnetism requires a modification of the $\mathrm{PBC}$ approach for $3 \mathrm{~d}$ periodic systems, as taking the infinite size limit leaves essential information about the shape of the (now 2d) surface unspecified which here matters for long-range interactions (see also discussion in section 3 in [1]).

To show why the shape matters, we consider two different structures $s_{\mathrm{A}}$ and $s_{\mathrm{B}}$ made of one billion individual cells each: $s_{\mathrm{A}}$ being a cube of $1000 \times 1000 \times 1000$ cells, and $s_{\mathrm{B}}$ being a film of $10000 \times 10000 \times 10$ cells. If both structures are homogeneously magnetized, a cell in the center of each experiences a demagnetizing field $\vec{H}=-T \vec{M}$. The demagnetizing tensor $T$ must satisfy (a consequence of the Maxwell equation $\operatorname{div} \vec{B}=0): \operatorname{tr} T=1$. For $s_{\mathrm{A}}$, we have $T^{(A)}=\operatorname{diag}(1 / 3,1 / 3,1 / 3)$, while for $s_{\mathrm{B}}$, we get $T^{(B)}=\operatorname{diag}(0,0,1)$. The form of $T$ depends on the shape of the far-away boundaries, but (for very large samples) not on the sample size.

In the macro geometry approach (which has been implemented in Nmag [2]), this issue is resolved by supporting the periodicity approximation such that the user specifies the placement of copies of the primary cell $C_{0}$. The physically most easily justifiable setups involve symmetric arrangements of the cells with $C_{0}$ at or close to the center. For the exchange field, the periodicity approximation is handled as usual for PBC.

The 'macro geometry' approach presented here - originally developed to address the necessity of handling the demagnetizing field correctly for $3 \mathrm{~d}$ PBC and used first in [3] - turns out to also be well suited for the study of small repetitive magnetic structures.

Macro geometry implementation The Nmag package [2] uses the hybrid finite element/boundary element method to compute the demagnetisation field [4] efficiently (see e.g. [5] for an overview). Conceptually, the boundary element matrix (BEM) captures the effects of the material surface. The entry in row $i$ and column $j$ of the matrix represents the contribution associated with surface node $i$ to surface node $j$.

For the macro geometry approach, the calculation of the BEM needs to be modified: In addition to the interaction of surface node $i$ with $j$ in the primary cell, we have to add the interaction of $j$ in the primary cell with $i$ in all image cells. Consequently, if $n$ image cells are used, the time to compute the BEM matrix will increase by a factor $n$. However, this does only affect the simulation setup time needed to compute the BEM. This behaviour parallels the PBC extension [1] for OOMMF [6].

The macro geometry approach can also be used in finite difference computations of the demagnetisation field.

Example 1 We start with a trivial simulation example to illustrate and validate the macro geometry idea. We consider a macro geometry of a rod of $135 \mathrm{~nm} \times 15 \mathrm{~nm} \times 15 \mathrm{~nm}$. We assume a uniform magnetisation throughout the geometry which we initially align with the $z$-axis, and compute the ratio of demagnetisation field $H_{\mathrm{d}}$ to saturation magnetisation $M_{\mathrm{s}}$ at the 
(A)

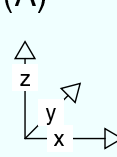

macro geometry

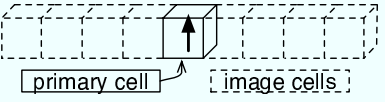

full system

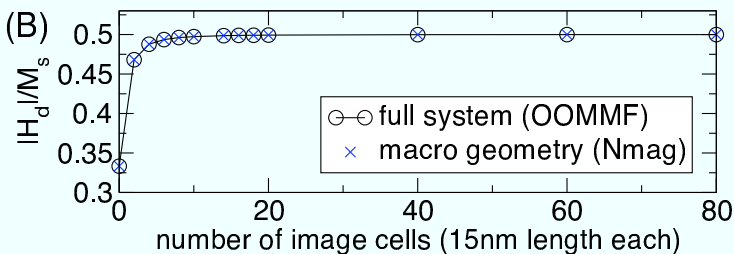

FIG. 1: (A): The rod geometry. (B): Demagnetising field $\left|H_{\mathrm{d}}\right| / M_{\mathrm{S}}$ in the center of the rod. We have used cubic cells with edge length $1 \mathrm{~nm}$ for the OOMMF calculations, and an unstructured mesh with an average edge length of $0.9 \mathrm{~nm}$ for the Nmag calculations.

center.

Within the macro geometry approach we model the system by a primary cell of one $(15 \mathrm{~nm})^{3}$ cube located centrally between 8 image cells as shown in Fig. 1A. We compare this with the simulation of the full $135 \mathrm{~nm}$ rod (with OOMMF), and obtain the same $z$-component of $H_{\mathrm{d}}$ at the center of the $\operatorname{rod}\left(\sim 0.496 M_{\mathrm{s}}\right)$.

Figure $1(\mathrm{~B})$ shows this ratio $\left|H_{\mathrm{d}}\right| / M_{\mathrm{S}}$ as a function of the rod length expressed through the number of image cells. There is excellent agreement between the micromagnetic calculation of the full rod and the macro geometry approximation. We get similar agreement (data not shown) for calculations with an uniform magnetisation pointing in the $x$-direction.

For this particular geometry and uniform magnetisation, the asymptotic values of the demagnetisation field (for infinite rod length) show the demagnetising factors of a magnetic needle, i.e. 0 for the magnetisation parallel to $x$ and $1 / 2$ for the magnetisation parallel to $z$ (or $y$ ).

The use of PBC, with an infinite number of image cells, corresponds to infinite rod length. Indeed using a PBC extension [1] for OOMMF with a cubic primary cell of $(15 \mathrm{~nm})^{3}$, we obtain the correct asymptotic values 0 and $1 / 2$ with an error of less than $10^{-8}$ (i.e. practically zero).

Example 2 Having demonstrated in the previous section that the macro geometry approach can correctly compute the demagnetisation field for a relatively trivial system, we now study a more realistic [7] quasi-periodic system shown in Fig. 2A.

First, we compute the micromagnetic dynamics of a chain of 8 spheres of diameter $50 \mathrm{~nm}$ each shown in Fig. 2B (i), before studying the full system of $31 \times 8$ spheres in example 3 . This sphere diameter is large enough to provide complicated vortex states in each sphere and small enough to allow the simulation of the full 8 sphere system in OOMMF (with cell size $1 \mathrm{~nm}^{3}$ ).

The initial configuration of the magnetisation $\left(M_{\mathrm{s}}=\right.$
(A)

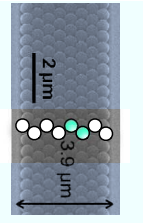

(B)

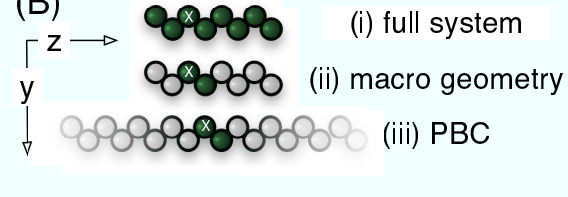

(C)

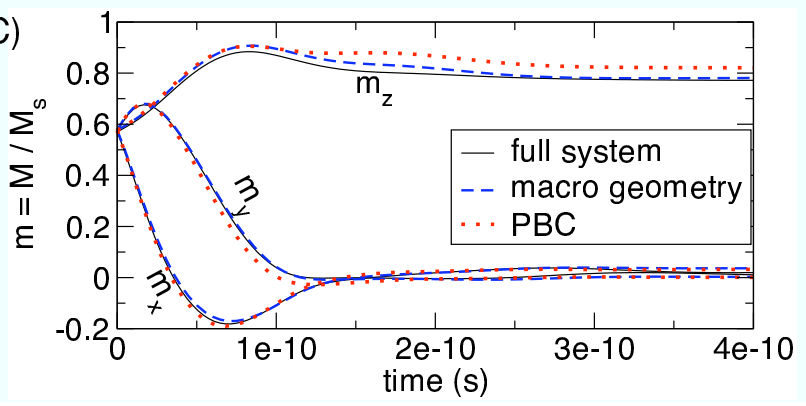

FIG. 2: $(A)$ : Latex template used for double template method [7] of sample growth. The total structure comprises $31 \times 8$ spheres (example 3 ) and the overlaid circles represent the arrangement of $1 \times 8$ ferromagnetic spheres studied in example 2 . (B): Schematic view of computational methods: (i) "full system" takes into account all 8 spheres (OOMMF). (ii) "macro geometry" has a primary cell that contains 2 (dark) spheres and 3 image cells that contain 6 (light) spheres to imitate the shape of the full system (Nmag). (iii) "periodic boundary conditions" (PBC) use a primary cell with 2 (dark) spheres and an infinite number of image cells (using OOMMF's PBC extension). $(C)$ : the resulting magnetisation trajectories. The magnetisation shown has been spatially averaged over one sphere which is indicated by a white ' $x$ ' in (B).

$\left.10^{6} \mathrm{~A} / \mathrm{m}\right)$ is pointing in the $(1,1,1)$ direction. We advance the magnetisation over time until it relaxes into an equilibrium state, using a damping constant of $\alpha=0.5$ and the exchange coupling $A=1.3 \cdot 10^{11} \mathrm{~J} / \mathrm{m}$.

Fig. 2B (ii) shows how one can study the 8 sphere system using the macro geometry approach by only considering two (dark) spheres in the primary cell, and by representing the other 6 (light) spheres through 3 image cells. The 2 real spheres and the 6 virtual spheres in the image cells are arranged exactly as in the full 8 sphere structure, and this is the key idea of the macro geometry approach.

Because we have 4 primary cells in this experimentally motivated structure, we cannot position the primary cell symmetrically in the center. Fortunately, numerical experiments with a chain of 10 spheres indicate that our choice is permissible nevertheless.

Fig. 2(C) shows the damped dynamics of the normalised $x, y$ and $z$ components of the magnetisation for the full chain as a thin black line, and for the macro geometry solution as a dashed blue line. The evolution of the magnetisation is remarkably similar, despite the full solution being 4 times more expensive to compute.

The small deviations between the full solution and the 

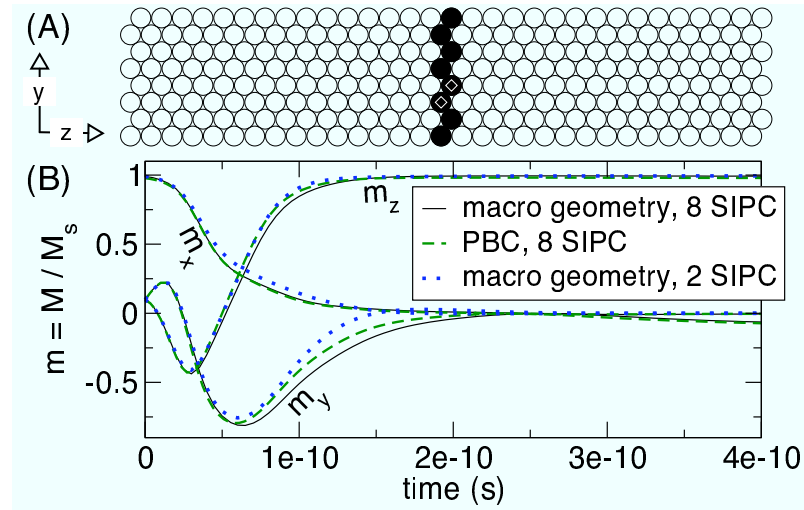

FIG. 3: $(A)$ : The array of $31 \times 8$ spheres under investigation. The macro geometry approach with "8 Spheres In Primary Cell" (8 SIPC) has a primary cell that includes the 8 filled spheres. All other spheres are represented trough 30 mirror images of this primary cell. The calculation with PBC has used the same primary cell but an infinite number of image cells to the left and right. The macro geometry approach with 2 spheres in the primary cell (2 SIPC) uses the 2 spheres marked with a white diamond. (B): The corresponding relaxation of magnetisation components for the three models.

macro geometry approximation come from (i) magnetisation configurations in the outer spheres that differ from magnetisation in the central spheres due to flux closure effects which cannot be captured by the macro geometry approximation, and from (ii) the different spatial discretizations used (finite elements for macro geometry and finite differences for full solution).

We also compute the magnetisation trajectory using OOMMF's PBC extension, for which we use 2 spheres in the primary cell (as in the macro geometry approach) and infinitely many image cells (in contrast to the macro geometry approach) as indicated in Fig. 2B (iii). This is the best approximation possible of the 8 sphere system using PBC. The PBC results are shown as a dotted red line in Fig. 2C, and also follow the full solution closely. Comparing the macro geometry results with the $\mathrm{PBC}$ results, we observe that the macro geometry approximation is closer to the full solution than the PBC. This is exactly the property we expect from the macro geometry approach for such samples of finite size: the shape effects of the overall (=macro) geometry of the sample should be captured through the demagnetisation field, and this information is lost when infinitely many image cells are used (unless the system is infinitely large, in which case the macro geometry and the PBC become equivalent).

Example 3 Finally, we compute the relaxation dynamics of the system of $31 \times 8$ spheres using three different methods which are demonstrated in Fig. 3A: (i) using the macro geometry with an 8 sphere primary cell, (ii) using PBC with the same primary cell, (iii) using the macro geometry with a small primary cell comprising only 2 spheres. The initial magnetisation is pointing in direc- tion $(1,0.1,0.1)$. The change of the coordinate system, relative to example 2, originates from OOMMF's PBC extension which requires the periodic dimension to be the $z$-axis.

Method (i), the macro geometry approach with an 8 sphere primary cell (solid black line in Fig. 3B), and method (ii), the PBC calculation, (dashed green line) are in relatively good agreement, and have the same computational effort of simulating the micromagnetics of 8 spheres. This similarity indicates that the overall shape of the $31 \times 8$ spheres structure is sufficiently slender to be approximated by a $\infty \times 8$ spheres structure as done by the PBC approach. While we do not know the correct micromagnetic solution for the $31 \times 8$ spheres structure, one could expect that the macro geometry approximation is more accurate than the $\mathrm{PBC}$ approximation (based on the results of Fig. 2C).

Because the macro geometry allows to place image cells at arbitrary positions, we can use method (iii): a primary cell with only 2 spheres (indicated by diamonds in Fig. $3 \mathrm{~A}$ ) to study the $31 \times 8$ sphere system (dotted blue line in Fig. 3B). In contrast to methods (i) and (ii), this smaller primary cell with only 2 spheres cannot resolve surface effects that take place in sphere 1 and sphere 8 of the primary cell, and a stronger deviation is thus expected. However, method (iii) is four times more efficient as it requires to solve the micromagnetic problem with 2 spheres only. This may be of substantial benefit in situations where some loss of accuracy is acceptable to make a system tractable for the first time.

Summary We have introduced a new approach which (i) improves modelling accuracy of small and large repetitive systems and (ii) for the first time allows the application of $3 \mathrm{~d}$ periodic boundary conditions in micromagnetism. We acknowledge financial support from EPSRC (EP/E040063/1,EP/E039944/1).

[1] K. M. Lebecki, M. J. Donahue, and M. W. Gutowski, J. Phys. D: Appl. Phys. 41, 175005 (2008).

[2] T. Fischbacher, M. Franchin, G. Bordignon, and H. Fangohr, IEEE Transactions on Magnetics 43, 2896 (2007), online at http://nmag.soton.ac.uk.

[3] G. Bordignon, T. Fischbacher, M. Franchin, J. P. Zimmermann, P. A. J. de Groot, and H. Fangohr, J.Appl.Phys. p. 07D932 (2008).

[4] D. R. Fredkin and T. R. Koehler, IEEE Trans. Magn. 26, 415 (1990).

[5] J. Fidler and T. Schrefl, Journal of Physics D: Applied Physics 33, R135 (2000).

6] M. J. Donahue and D. G. Porter, OOMMF User's Guide, NIST, Gaithersburg, MD (1999), interagency Report NISTIR 6376.

[7] M. A. Ghanem, P. N. Bartlett, P. A. J. de Groot, and A. Zhukov, Electrochemistry Comm. 6, 447 (2004). 